\title{
APPLICATION OF INTELLECTUAL MONITORING INFORMATION TECHNOLOGY IN DETERMINING THE SEVERITY OF THE CONDITION OF PATIENTS WITH INFLAMMATORY BOWEL DISEASES
}

D0I: $10.36740 /$ WLek202103118

\author{
Andriy E. Dorofeyev' ${ }^{1}$, Sergiy V. Holub' ${ }^{2}$, Gulustan H. Babayeva ${ }^{3}$, Oleg E. Ananiin ${ }^{2}$ \\ 'INTERNAL DISEASES, SHUPYK NATIONAL MEDICAL ACADEMY OF POSTGRADUATE EDUCATION, KYIV, UKRAINE \\ ${ }^{2}$ COMPUTER TECHNOLOGY, CHERKASY STATE TECHNOLOGICAL UNIVERSITY, CHERKASY, UKRAINE \\ ${ }^{3}$ DEPARTMENT OF THERAPY, AZERBAIJAN STATE ADVANCED TRAINING INSTITUTE FOR DOCTORS NAMED AFTER A.ALIYEV, BAKU, AZERBAIJAN
}

\begin{abstract}
The aim: Was to evaluate the effectiveness of the use of information technology of intelligent monitoring in solving the problems of assessing the morbidity of a patient with IBD during treatment.

Matherials and methods: 183 patients with IBD were observed. Among them 104(56.8\%) patients suffered from Crohn's disease and 79(43.1\%) patients had ulcerative colitis. For each patient and each disease, the formation of a list of signs, the extraction of information and knowledge will be carried out according to an individual method. At the lower level, tasks are performed: determination of the list of patient morbidity conditions, the formation of a list of indicators of the patient morbidity conditions, their identification as classes for machine learning models; formation of a list of signs, which identify the state of the patient's morbidity and whose characteristics obtained after results of medical tests. Results: The number of correctly classified points reached $92 \%$. An analysis of the conditions of patients characterized by incorrectly classified points revealed the information content of this fact. In those cases when the classification results did not coincide with the expert assessment of the patient's condition, additional factors were found that influenced his condition and whose characteristics were not taken into account in the structure of classifier models.

Conclusions: The results of the testing of classifier models indicate the effectiveness of the use of information technology of intelligent monitoring to assess the condition of patients with IBD.
\end{abstract}

KEY WORDS: Intellectual monitoring, information technology, inflammatory bowel diseases

Wiad Lek. 2021;74(3 p.l):481-486

\section{INTRODUCTION}

One of the main problems in monitoring inflammatory bowel diseases (IBD) is the high level of uncertainty in the causes of these pathologies. This is due to the physician's limited ability to study the development processes of this pathology in various classes of patients and a significant level of individuality in the processes of disease formation and the course of the disease. The expansion of the doctor's capabilities is achieved today by applying data processing technologies, in particular modeling [1]. The simulation results largely depend on the understanding by the designer of the subject area, knowledge of the technologies for the formation of the initial description of the object and the formation of a database with the results of observations, the successful use of methods for constructing models, the correct application of the model testing process and the interpretation of modeling results.

It is also assumed that cost software is available that can adapt to changing the individual properties of each patient. A distinctive feature of this study is that its authors use intelligent monitoring technology as a whole, rather than modeling an individual element, to achieve their goals. In a single process of generating input data arrays (IDA), expert doctors, scientists, analysts, and fashion designers with intelligent monitoring technologies are combined. A monitoring intelligent system is also used - a software package that implements the process of obtaining information from observation results, turning it into a knowledge base and using this database as an algorithm for converting new data $[2,3]$.

The synthesis of individual models is carried out on the basis of the initial description obtained from the results of observations of a group of patients - determining the characteristics of the patient's condition by conducting medical tests at different stages of the disease. The primary description is formed by recording the numerous characteristics of significant factors, a list of which is formed by an expert doctor [4].

The indisputable advantage of ITIM is that the monitoring technology is built for each external order individually for each decision-making process. That is, the formation of a sequence of stages of observation is technologically 
ensured to build a base of model knowledge about the disease process of each patient individually [5].

\section{THE AIM}

The aim of the study is to evaluate the effectiveness of the use of information technology of intelligent monitoring in solving the problems of assessing the morbidity of a patient with IBD during treatment.

It is required to solve the problem of classifying the patient's conditions when there is a classification of observation points for a patient with IBD in a multidimensional space of signs $\mathrm{X}$ results of medical tests. It is necessary to build the rules for the reflection of the set of signs of $\mathrm{X}$ to the set of classes $\mathrm{P}$ (determination of disease states)

The results of this task are used to create a non-invasive method in the diagnosis of the patient's morbidity by processing the results of standard medical tests and machine learning models, without painful procedures and complications after diagnosis.

\section{MATERIALS AND METHODS}

183 patients with IBD were observed. Among them 104(56.8\%) patients suffered from Crohn's disease and 79(43.1\%) patients had ulcerative colitis. All patients population was aged from 17 to 60 years. Duration of IBD were 1.2-9.4 years. 20 patients with a diagnosis of irritable bowel syndrome and 20 healthy persons were in control group.

In all patients complaints, clinical picture of the disease were analyzed before and after treatment, during 3 years of observations. Harvey Bradshaw CD Activity Index for Crohn's disease patients, Truelove-Witts criteria and Mayo Index for ulcerative colitis patients were used [6].

Laboratory parameters were studied. In all IBD patients and in control group levels of highly sensitive CRP, homocysteine, platelets, vitamin $\mathrm{D}$, fecal calprotectin and albumin in the urine were observed (table I). During observation period all IBD patients obtained treatment according to protocols of European Crohn's and Colitis Organisation.

For each patient and each disease, the formation of a list of signs, the extraction of information and knowledge will be carried out according to an individual method.

According to the methodology for creating information technologies of multi-level intelligent monitoring [7], each local monitoring task is solved by constructing a separate model, a set of models of one level of information conversion are formed by strata, strata are combined into a global functional dependence. The combination of global functional dependencies forms the base of model knowledge [8]. The base of model knowledge is included in the structure of intelligent agents and multi-agent systems and determines their functioning as intended. Based on multi-agent systems, the information technology of multi-level intelligent monitoring is being built [2]. In accordance with the objectives of this work, two levels of information conversion were identified. At thelower level, the fulfillment of the complex of tasks is aimed at ensuring the reflection of the elements of the set of results of analyzes of $X$ to the elements of the set of indicators of the state of the patient Y. At the upper level, it is necessary to ensure the grouping of the elements of the set of indicators of the conditions into classes of the patient's incidence. The constructed models solve the problems of transforming information of one level, form a stratum.

At the lower level, tasks are performed:

1) determination of the list of patient morbidity conditions, the formation of a list of indicators of the patient morbidity conditions of the set $Y=\{y 1, y 2, \ldots, y \mathrm{~m}\}$, their identification as classes for machine learning models;

2) the formation of a list of signs of the set $X=\{x 1, x$ $2, \ldots, \mathrm{x} \mathrm{n}\}$, which are sufficient to identify the state of the patient's morbidity and whose characteristics are planned to be obtained after tillage and the results of medical tests;

3) conducting research on patients. Standard procedures are carried out for the expert assessment of the patient's condition by the doctor using traditional methods of diagnosis, prescribing medical tests, processing their results, presenting the results in a standard form of a two-dimensional table of data for the initial description of the patient:

$\left(\begin{array}{cccccccc}x_{11} & x_{12} & \ldots & x_{1 n} & y_{11} & y_{12} & \ldots & y_{1 m} \\ x_{21} & x_{22} & \ldots & x_{2 n} & y_{21} & y_{22} & \ldots & y_{2 m} \\ \ldots & \ldots & \ldots & \ldots & \ldots & \ldots & \ldots & \ldots \\ x_{k 1} & x_{l 2} & \ldots & x_{k n} & y_{k 1} & y_{k 2} & \ldots & y_{k m}\end{array}\right)$

where xij is the $j$-th indicator of the patient's analysis of the $\mathrm{i}$-th observation, yij is the $\mathrm{j}$-th indicator of the patient's condition of the $\mathrm{i}$-th observation, $\mathrm{k}$ is the number of series of analysis of observations; $\mathrm{n}$ is the number of indicators planned to be obtained by the doctor according to the results of the analyzes, $\mathrm{m}$ is the number of characteristics of the patient's condition.

4) the construction of models that contain a solution to the problem of identifying the functional dependence of state indicators $\mathrm{Y}$ on the array of monitoring results (medical tests) $\mathrm{X}$ :

$y_{\mathrm{i}}=f\left(\mathrm{x}_{\mathrm{j}}\right), i=1, m ; j=1, n$,

where $n$ is the number of indicators planned to be obtained by the doctor according to the results of the analyzes, $\mathrm{m}$ is the number of characteristics of the patient's conditions.

At the top level of information conversion, the following tasks are performed:

1) study of the output signals of the lower stratum models, the formation of an input data array and the synthesis of the upper start models [3];

2) the synthesis of classifier models for grouping points (monitoring the patient's condition) according to the morbidity classes;

3 ) model testing and interpretation of classification results. The formulated classification problem is poorly formalized. Signs of belonging of the observation point to the class of disease states according to the results of medical tests cannot be clearly set without additional diagnostic procedures. Therefore, for each class of states, a classifier model is built. Models are built for each feature (there are rules for reflecting multiple features into multiple classes $\mathrm{P}$ ). After that, decision rules are built (the second level of monitoring), which determine the patient's belonging to one of the classes (conditions) by: 
1. Calculation of the value of one of the modeled indicators of the state of the set $Y=\{y 1, y 2, \ldots, y m\}$ by substituting the results of medical tests from the set $X=\{x 1, x 2, \ldots$, $\mathrm{xn}\}$. The vector of values of the results of the analysis is converted into the numerical value of the sign of the patient's condition.

2. The formation of an array of input data (MVD) for the stratum of the second level. Models of the second-level stratum contain solutions to the classification problem: construct a resolving rule a, which will correctly identify the new state of the patient (3):

$Z_{i}=f\left(y_{i}\right), i=1, k ; j=1, m$

to one of the described classes $P=\left\{\mathrm{p}_{1}, \mathrm{p}_{2}, \ldots \mathrm{p}_{1}\right\}$ : a: $Z \rightarrow P$

A model is adequate if, according to the results of its use, a significant number of patient conditions are correctly classified. A significant number of correctly classified states that allow us to consider the model adequate is set by an expert method. The model is stable in the case when it is possible to provide the specified indicators of adequacy on the examination sequence of the patient's conditions, the characteristics of which were not used in the process of model building. If the model is adequate and stable, then this means that the structure of the patient's body is correctly reflected in its structure, that is, the model contains information. In this case, the results of the assessment of the influence of factors [3] will be reliable.
Adequacy and sustainability are characteristics of the utility of the model. A model is considered useful when, based on the results of its use, information about the patient is obtained, which allows the doctor to achieve the patient's recovery, or at least to improve his condition.

Simulation processes are used to transform data in monitoring intelligent systems. The monitoring system is built to provide information to decision-making processes in a given subject area. The decision maker determines the consequences of applying each of the available strategies.

In conditions of medical monitoring, the person making the decision is a doctor, strategies are treatment regimens, the object of monitoring is the patient's condition.

The purpose of medical monitoring is to provide the doctor with information about the patient's condition, that is, the diagnosis, about the properties of the body in the fight against the disease, about the mechanisms of disease formation and to predict the change in the patient's condition when applying one or another treatment regimen.

\section{RESULTS}

To accomplish the tasks, the following hypothesis was formulated: "Determining the condition of a patient with IBD is possible by constructing classifier models with an intelligent monitoring information system and expert

Table I. The list of features that were used to form the primary description

\begin{tabular}{|c|c|c|c|}
\hline $\begin{array}{c}\text { № } \\
\text { in order }\end{array}$ & sign & a comment & variable \\
\hline 1 & $\begin{array}{l}\text { Highly sensitive } \\
\text { CRP, mg / L }\end{array}$ & $\begin{array}{l}\text { C-reactive protein, highly sensitive method (h / sCRP) - a study that reveals a slight } \\
\text { excess concentration of C-reactive protein. In general, an increase in the level of } \\
\text { C-reactive protein (CRP) with high sensitivity indicates an inflammatory process that } \\
\text { occurs in the body }[9,10] \text {. }\end{array}$ & $x_{1}$ \\
\hline 2 & $\begin{array}{l}\text { Homocysteine, } \\
\mu \mathrm{mol} / \mathrm{L}\end{array}$ & $\begin{array}{l}\text { One of the markers of endothelial dysfunction is a change in the level of homocysteine, } \\
\text { which is a replaceable cytotoxic and neurotoxic amino acid, which is present in } \\
\text { all cells of the body and plasma in small quantities. data indicate that the level of } \\
\text { homocysteinemia can serve as an independent marker of pathogenetic changes in IBD } \\
{[6,9,10] .}\end{array}$ & $x_{2}$ \\
\hline 3 & $\begin{array}{l}\text { Platelets, } \\
\text { thousand } / \mathrm{mm}^{3}\end{array}$ & $\begin{array}{l}\text { Blood cell characteristics, but in this study are considered as a marker of inflammation } \\
\text { activity. With their increase, the risk of blood clots increases against the background of } \\
\text { the damaging effect of homocysteine on the inner shell, vascular intima, thrombosis } \\
\text { of small vessels may develop with a decrease in oxygen supply to tissues, which is } \\
\text { important in conditions of relative energy deficiency that occurs with inflammation }\end{array}$ & $x_{3}$ \\
\hline 4 & $\begin{array}{l}\text { Vitamin D, } \\
\mathrm{ng} / \mathrm{mL}\end{array}$ & $\begin{array}{l}\text { A group of biologically active substances (including cholecalciferol and ergocalciferol). } \\
\text { The main function of both cholecalciferol and ergocalciferol is to ensure the absorption } \\
\text { of calcium and phosphorus from food in the small intestine. We can also assume } \\
\text { the following additional functions of vitamin D: participation in the regulation of } \\
\text { cell reproduction, metabolic processes, stimulation of the synthesis of a number of } \\
\text { hormones [10] }\end{array}$ & $x_{4}$ \\
\hline 5 & $\begin{array}{l}\text { Calprotectin } \\
\text { fecal, } \mu \mathrm{g} / \mathrm{g}\end{array}$ & $\begin{array}{l}\text { A protein produced by neutrophils in the intestinal mucosa. Its level (in feces) is } \\
\text { increased in Crohn's disease and ulcerative colitis, in addition, this indicator is increased } \\
\text { in infectious bowel infections, cancer, in remission phase }[6,10] \text {. }\end{array}$ & $x_{5}$ \\
\hline 6 & $\begin{array}{l}\text { Albumin in the } \\
\text { urine, } \mathrm{mg} / \mathrm{L}\end{array}$ & $\begin{array}{l}\text { The main plasma protein, its purpose is the construction of new cells in the form of } \\
\text { essential amino acids. Microalbuminuria - the allocation of albumin in the urine in } \\
\text { an amount of 30-300 mg per day; refers to the early signs of impaired renal function } \\
\text { and is one of the manifestations of target organ damage (an indicator of endothelial } \\
\text { dysfunction, insulin resistance and hypercoagulation) [9]. }\end{array}$ & $x_{6}$ \\
\hline
\end{tabular}


interpretation of modeling results."

For each patient and each disease, the formation of a list of signs, the extraction of information and knowledge will be carried out according to an individual method. Modeling was carried out according to the results of observations obtained from August 2015 to December 2018 at the Therapy Department of the Azerbaijan State Institute of Advanced Medical Training named after A. Aliyev. We studied 183 patients with Crohn's disease (56.8\%) and ulcerative colitis (43.1\%) aged 17 to 60 years with a disease duration of 1.2-9.4 years. Verification of the results was provided by introducing 20 patients with a diagnosis of Irritable Bowel Syndrome and 20 people into the studies, who have no complaints.

To form an AID, the features described in Table I were expertly determined.

Characteristics of the characteristics presented in table I were obtained by conducting medical tests according to standard methods. If necessary, the tests were repeated, a total of 426 cases. Two groups of patients were formed (A and B), commensurate in gender and age. The feature vectors constructed from the results of group A observations formed an AID for training models. The results of observations of group B were used to test trained models.

The results of the expert classification of the patient's condition were used as a simulated indicator. It was suggested to distinguish four classes (states) of the patient -0 (clinical and endoscopic remission); 1 (mild course of the disease); 2 (medium severity); 3 (serious condition of the patient). Table II presents the characteristics of the classes. Characteristics of the condition of the patients were analyzed by IBD activity indexes [6]. These characteristics fulfill the function of classification features. Clinical and endoscopic remission is characterized by the absence of clinical manifestations and macroscopic changes during endoscopy. The severity of the disease as a whole is determined by: the severity of the current attack, the presence of extraintestinal manifestations and complications, refractory treatment, in particular, the development of hormonal dependence and resistance [6]. The severity of the disease as a whole is determined by: the severity of the current attack, the presence of extraintestinal manifestations and complications, refractory treatment, in particular, the development of hormonal dependence and resistance [6].

The model was synthesized by a classifier, which was designed based on the agent approach [7]. Each agent used different methods for building the model. An agent was selected that provided the best classification results.

A group of the best models according to the quality criterion was chosen to build the classifier. The criterion for the quality of models is the number of correctly classified observation points on the examination sequence in a multidimensional space of features of the primary description. Each observation point describes an experimentally determined condition of the patient in accordance with table II.

The observation point in the multi-factorial space of signs is a vector that contains a numerical characteristic - the class value and the values of the numerous charac- teristics of the characteristics presented in Table I. The set of observation points forms AID in the form (1). Table III presents the test results of classifier models on a sequence of observation points $B$.

The number of correctly classified points reached $92 \%$. An analysis of the conditions of patients characterized by incorrectly classified points revealed the information content of this fact. In those cases when the classification results did not coincide with the expert assessment of the patient's condition, additional factors were found that influenced his condition and whose characteristics were not taken into account in the structure of classifier models.

\section{DISCUSSION}

Information technology of multilevel intellectual monitoring [3] is used to study objects classified as complex systems in the conditions of low information content of their primary description. The object of study is reflected in the form of a complex system in the case when, in order to obtain its adequate model, it is necessary to use information that is not yet available [4]. Additional information about the properties of the monitoring object is acquired in the process of continuous observation, processing and conversion of their results [3]. One of the monitoring needs in the medical industry is to identify the mechanisms of disease formation and determine the condition of the patient according to the results of certain observations. The purpose of observations, as a rule, is to form an array of numerous characteristics that will allow, in the process of building the model, to reflect in its structure the properties of the patient's body to respond to control influences that are part of the treatment regimen. In [1], to obtain numerous characteristics, the surface of the colon was photographed during endoscopy. As a result of these observations, a number of images were obtained. According to the results of observations, the task of classifying the patient's disease was solved. Three classes have been described: 1) the patient has Crohn's disease; 2) the patient has ulcerative colitis 3 ) the patient is conditionally healthy and there are no signs of Crohn's disease and ulcerative colitis. The obtained images were segmented, a list of attributes of each segment was determined, an array of numerous characteristics of these signs was formed. To classify the images, a resolution rule was constructed in the form of artificial neural networks based on a multilayer perceptron. It was possible to obtain $84.2 \%$ of correctly classified images.

In [5], an array of input data was formed based on the results of observations carried out in the form of spectral analysis of the blood serum of patients. As in the previous case, three classes of patients were described: with Crohn's disease, with ulcerative colitis and conditionally healthy. The observation results were obtained in the form of spectrograms with characteristic peaks. The characteristics of the peaks and their placement on the spectrogram are used as numerical characteristics of the observation results. An array of numerous characteristics was subjected to multivariate entropy analysis using the DiaStat statistical 
Table II. Description a class states of patients

\begin{tabular}{ccc}
\hline Classes & Title & The value of the class for modeling \\
\hline 0 & Clinical and endoscopic remission & 0 \\
\hline 1 & Easy course of the disease & 50 \\
\hline 2 & Medium severity condition & 500 \\
\hline 3 & Severe condition of the patient & 1000 \\
\hline
\end{tabular}

Table III. Test results of classifier models

\begin{tabular}{|c|c|c|c|c|c|c|c|c|c|c|c|c|c|c|c|}
\hline 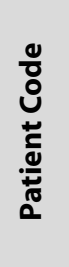 & 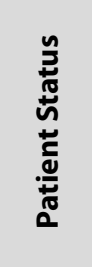 & 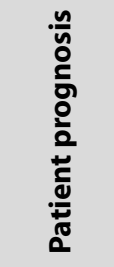 & 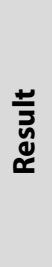 & 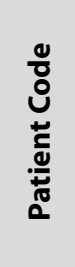 & 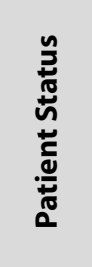 & 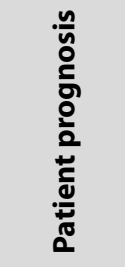 & 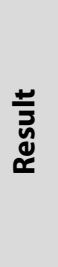 & 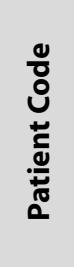 & 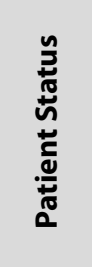 & 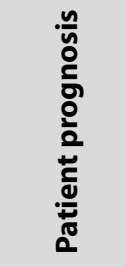 & 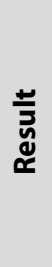 & 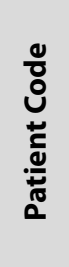 & 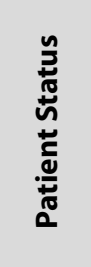 & 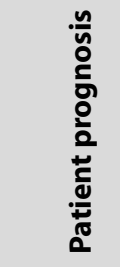 & 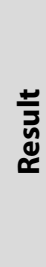 \\
\hline 251 & 1000 & 999,61 & 1 & 220 & 50 & 50,85 & 1 & 189 & 500 & 390,26 & 1 & 158 & 1 & 1,077 & 1 \\
\hline 250 & 1 & 0,36 & 1 & 219 & 500 & 499,96 & 1 & 188 & 500 & 387,20 & 1 & 157 & 1 & 0,939 & 1 \\
\hline 249 & 1 & 6,80 & 1 & 218 & 50 & 72,09 & 1 & 187 & 50 & 100,75 & 1 & 156 & 1 & 9,56 & 1 \\
\hline 248 & 1 & 1,58 & 1 & 217 & 50 & 50,04 & 1 & 186 & 1000 & 100,75 & 0 & 155 & 1 & 3,2 & 1 \\
\hline 247 & 1 & 47,61 & 0 & 216 & 50 & 49,43 & 1 & 185 & 50 & 151,56 & 1 & 154 & 1 & 0,34 & 1 \\
\hline 246 & 50 & 133,07 & 1 & 215 & 500 & 420,30 & 1 & 184 & 500 & 499,13 & 1 & 153 & 1 & 6,37 & 1 \\
\hline 245 & 50 & 160,18 & 1 & 214 & 500 & 350,53 & 1 & 183 & 1 & 1,005 & 1 & 152 & 1 & 103,51 & 0 \\
\hline 244 & 500 & 411,96 & 1 & 213 & 50 & 65,63 & 1 & 182 & 50 & 150,38 & 1 & 151 & 1000 & 933,28 & 1 \\
\hline 243 & 50 & 143,03 & 1 & 212 & 1 & 0,007 & 1 & 181 & 50 & 71,77 & 1 & 150 & 1 & 57,18 & 0 \\
\hline 242 & 500 & 497,25 & 1 & 211 & 50 & 50,48 & 1 & 180 & 50 & 52,73 & 1 & 149 & 1000 & 998,94 & 1 \\
\hline 241 & 1 & 0,66 & 1 & 210 & 500 & 397,37 & 1 & 179 & 1 & 6,93 & 1 & 148 & 1 & 0,68 & 1 \\
\hline 240 & 50 & 50,00 & 1 & 209 & 500 & 318,44 & 1 & 178 & 50 & 49,83 & 1 & 147 & 1 & 34,82 & 1 \\
\hline 239 & 50 & 49,02 & 1 & 208 & 1000 & 626,10 & 0 & 177 & 1 & 2,66 & 1 & 146 & 1 & 0,58 & 1 \\
\hline 238 & 50 & 65,80 & 1 & 207 & 1000 & 1035,35 & 1 & 176 & 500 & 499,87 & 1 & 145 & 1000 & 1001,57 & 1 \\
\hline 237 & 500 & 290,48 & 1 & 206 & 1000 & 1006,36 & 1 & 175 & 500 & 494,14 & 1 & 144 & 1 & 0,67 & 1 \\
\hline 236 & 50 & 50,00 & 1 & 205 & 500 & 361,09 & 1 & 174 & 1000 & 1000,3 & 1 & 143 & 1 & 147,14 & 0 \\
\hline 235 & 1 & 0,23 & 1 & 204 & 500 & 333,27 & 1 & 173 & 1000 & 727,46 & 1 & 142 & 1 & 1,01 & 1 \\
\hline 234 & 500 & 349,57 & 1 & 203 & 500 & 499,37 & 1 & 172 & 50 & 50,21 & 1 & 141 & 1000 & 998,07 & 1 \\
\hline 233 & 1000 & 904,35 & 1 & 202 & 50 & 166,76 & 1 & 171 & 1 & 1,004 & 1 & 140 & 50 & 50,16 & 1 \\
\hline 232 & 500 & 480,55 & 1 & 201 & 500 & 344,90 & 1 & 170 & 1 & 50,75 & 0 & 139 & 500 & 384,69 & 1 \\
\hline 231 & 50 & 126,36 & 1 & 200 & 1 & 1,92 & 1 & 169 & 1 & 1,64 & 1 & 138 & 50 & 77,25 & 1 \\
\hline 230 & 500 & 503,33 & 1 & 199 & 50 & 79,68 & 1 & 168 & 1 & 1,014 & 1 & 137 & 50 & 79,39 & 1 \\
\hline 229 & 500 & 499,93 & 1 & 198 & 1 & 47,91 & 0 & 167 & 1000 & 1069,86 & 1 & 136 & 500 & 486,89 & 1 \\
\hline 228 & 500 & 287,16 & 1 & 197 & 500 & 498,03 & 1 & 166 & 500 & 515,97 & 1 & 135 & 50 & 55,69 & 1 \\
\hline 227 & 1 & 0,74 & 1 & 196 & 500 & 252,61 & 1 & 165 & 500 & 500,45 & 1 & 134 & 1 & 4,97 & 1 \\
\hline 226 & 1 & 17,34 & 1 & 195 & 50 & 50,73 & 1 & 164 & 500 & 499,53 & 1 & 133 & 1 & 0,43 & 1 \\
\hline 225 & 1 & 0,06 & 1 & 194 & 1 & 2,02 & 1 & 163 & 500 & 487,76 & 1 & 132 & 50 & 50,37 & 1 \\
\hline 224 & 500 & 495,87 & 1 & 193 & 1 & 1,06 & 1 & 162 & 50 & 56,5 & 1 & 131 & 50 & 48,80 & 1 \\
\hline 223 & 50 & 49,42 & 1 & 192 & 50 & 109,59 & 1 & 161 & 500 & 451,12 & 1 & 130 & 1 & 93,66 & 0 \\
\hline 222 & 500 & 457,39 & 1 & 191 & 1 & 4,28 & 1 & 160 & 1 & 286,76 & 0 & & & & \\
\hline 221 & 1 & 12,10 & 1 & 190 & 50 & 50,31 & 1 & 159 & 1 & 1,029 & 1 & & & & \\
\hline
\end{tabular}


analysis software package [7]. According to the authors, they managed to get $93-95 \%$ of correctly classified objects. It was not possible to detect methods for non-invasive assessment of the dynamics of the pathology in a patient with IBD, classification of his condition.

Today, the task of creating a reliable methodology for non-invasive diagnosis of a condition in IBD remains relevant. The results of the classification of diseases described in the literature require improvement, bringing the process of obtaining individual scientific results to the level of technology that is available to the ordinary doctor. The process of using the simulation results in selecting a treatment regimen requires additional research. It is also necessary to predict the individual response of the patient to the standard treatment regimen.

\section{CONCLUSIONS}

The results of the testing of classifier models indicate the effectiveness of the use of information technology of intelligent monitoring to assess the condition of patients with IBD. According to the results of standard medical tests, presented in the form of a two-dimensional array of numerous characteristics, the doctor has the opportunity to assess the individual response of the patient to the treatment regimen and identify the need for its correction.

\section{REFERENCES}

1. Bakulin I., Skalinskaya M., Skazyvaeva E. et al. Artificial neural network as an assistant in the differential diagnosis between ulcerative colitis and Crohn's disease. United European Gastroenterology Journal. 2019;7:336-337

2. HolubS., Kunytska S. The concept of multi-agent intellectual monitoring systems Projekt interdyscyplinarny projektem XXI wieku. Processing, transmission and security of information. 2019: 183-188.

3. Holub S.V. Multilevel modeling in environmental monitoring technologies. Bohdan Khmelnytsky National University. 2007: 220.

4. Peregudov F.I., Tarasenko F.P. Introduction to systems analysis. Higher school. 1989: 367.

5. Fedulova E.N., Gordetsov A.S., Fedorova A.V. et al. Using a mathematical model of infrared spectroscopy of blood serum in the differential diagnosis of ulcerative colitis and Crohn's disease in children. Bulletin of the Russian Academy of Medical Sciences. 2013;12: 44-48.
6. Chen J.H., Andrews J.M., Kariyawasam V., Moran N. IBD Sydney Organisation and the Australian Inflammatory Bowel Diseases Consensus Working Group. Review article: acute severe ulcerative colitis - evidence-based consensus statements. Aliment Pharmacol Ther. 2016;44(2):127-44.

7. Kunytska S., Holub S. Multi-agent Monitoring Information Systems. Mathematical Modeling and Simulation of Systems . MODS 2019 Advances in Intelligent Systems and Computing, Springer, Cham. 2019; 1019: 164-171.

8. Zhiryakova I.A., Holub S.V. New approach to conceptual knowledge. Technical science and technology, 2015; 2; 78-82.

9. Babayeva G. H., Babayev Z. M. A new approach to assessing the clinical condition of patients with ulcerative colitis and Crohn's disease. Experimental and clinical gastroenterology. 2019; 162 (2): 19-23.

10. Babayeva G.H., Babayev Z.M. The frequency of detection of some markers of endothelial dysfunction in patients with inflammatory bowel disease. Therapeutic archive. 2018; 4: 12-16.

\section{ORCID and contributionship:}

Andriy E. Dorofeyev: 0000-0002-2631-8733 A,B,E,F

Sergiy V. Holub: 0000-0002-5523-6120 ${ }^{A, D, F}$

Gulustan H. Babayeva: 0000-0002-5805-3741 B,D,E

Oleg E. Ananiin: 0000-0001-9203-597X B,C

\section{Conflict of interest:}

The Authors declare no conflict of interest.

\section{CORRESPONDING AUTHOR}

\section{Andriy E. Dorofeyev}

Shupyk national medical academy of postgraduate education

9 Dorogozhitskaya st., 04112 Kiev, Ukraine

tel: +380997693945

e-mail:dorofeyevand@gmail.com

Received: 15.04 .2020

Accepted: 10.11 .2020

A - Work concept and design, B - Data collection and analysis, C - Responsibility for statistical analysis, D-Writing the article, $\mathbf{E}$-Critical review, $\mathbf{F}$ - Final approval of the article 